\title{
The use of pharmacological protocols during the Covid-19 crisis - a déjà vu of the
}

\section{A/H1N1 pandemic experience}

\author{
Wladimir Jimenez Alonso ${ }^{1 *}$ \\ Veronique Diez Vicera ${ }^{1}$ \\ Cynthia Schuck-Paim ${ }^{1}$
}

\section{ABSTRACT}

Only one decade ago, the World Health Organization declared the outbreak of a novel influenza A (H1N1) virus a worldwide pandemic. Then, just as in today's Covid-19 crisis, treatment options were the center of debate, one of which is of special importance to the current pandemic: the existence of pharmacological treatments that, although available and potentially effective, are approached with reluctance due to concerns around side effects, the development of resistance and lack of conclusive evidence of effectiveness from randomized-controlled trials. History has proven that reluctance to use antivirals during the $2009 \mathrm{~A} / \mathrm{H} 1 \mathrm{~N} 1$ pandemic was unwise, as those countries that restricted their use to at-risk groups and to patients with advanced disease experienced higher mortality. Those same antiviral are now routinely used for seasonal influenza. We revisit this forgotten lesson as a means of weighing in on the debate over the use of new treatments with promising outcomes as observed in clinical practice, but lacking strong evidence from controlled trials, which require a time frame incompatible with that available in unfolding public health emergencies triggered by novel pathogens.

\section{INTRODUCTION}

We must recognize the immense dilemma that physicians face during public health emergencies, particularly those triggered by novel pathogenic threats, when therapeutic interventions and other life-and-death decisions must be made with incomplete and conflicting information. On the one hand, history tends to highlight the (easily visible) damages of action, while hiding the (less visible, but not any less real) casualties of inaction. From the healthcare provider's perspective, the simpler choice would be to avoid the liabilities potentially brought about by the employment of new treatment approaches or by deviation from general recommendations. On the other hand, this course of action also bears multiple costs, as it hinders the use and development of potentially life-saving treatments at critical periods during unfolding emergencies, and downplays the costs of inaction, namely the risk of death, complications and prolonged used of healthcare resources when the disease is left untreated. A recurring theme in the current SARS-CoV-2 pandemic has been the reluctance towards the use of new therapeutic interventions (or the repositioning of existing drugs for novel therapeutic purposes) due to uncertainty about efficacy and concerns around potentially adverse effects. Often, even those drugs with well established safety profiles are used or advised only as a last treatment resource, at a time when recovery is less likely. In this discussion, proper balancing of the adverse effects of inaction in benefit-risk assessments of targeted drugs is often lacking. Additionally, advocacy for the need

\footnotetext{
${ }^{1}$ Epidemiology Research Group EPIDOT, Public Health Department, Federal University of Santa Catarina (UFSC), Santa Catarina, Brazil

*Corresponding author: Wladimir Jimenez Alonso. Address: Epidemiology Research Group EPIDOT, Public Health Department, Federal University of

Santa Catarina (UFSC), Florianópolis, Santa Catarina, 88040-970, Brazil. wladimir.j.alonso@gmail.com
}

Como citar este artigo / How to cite this article

Alonso WJ, Vicera VD, Schuck-Paim C. The use of pharmacological protocols during the Covid-19 crisis - a déjà vu of the 2009 A/H1N1 pandemic experience InterAm J Med Health 2020;3:e202003059. 
of efficacy evidence from randomized clinical trials as the only means of establishing benefit has been pervasive, even though these trials require a time frame incompatible with that available in public health emergencies.

In this piece, we provide a brief reminder of the illustrative experience with antiviral drugs during the 2009 A/H1N1 influenza pandemic. In that year, two effective neuraminidase inhibitors - oseltamivir and zanamivir - were widely available, as many governments had stockpiled them for years for use specifically in a potential influenza pandemic. Though several countries took advantage of this pharmacological resource, a long-lasting reluctance to utilize these antivirals by many others had tragic consequences. When a vaccine arrived, one year later, it was too late. Oseltamivir and zanamivir, which were particularly effective during the early symptomatic phase of infected patients, could have spared many of those who died from the $\mathrm{A} / \mathrm{H} 1 \mathrm{~N} 1$ virus, namely an estimated quarter of a million people [1]. Instead, doubt surrounding their potential side effects and lack of substantial efficacy data from controlled trials at the time fostered underutilization. The experience is emblematic of the lessons that should be learnt to prevent the same mistakes from being repeated in the management of today's Covid-19 crisis and future events.

\section{Sole reliance on clinical trial results for decision- making}

Over the course of the current pandemic, the notion of what constitutes scientific evidence has been often deflated to only one specific methodology, the randomized clinical trial. The belief that only this experimental paradigm can provide an acceptable degree of certainty about the efficacy of a therapy would be misleading even under normal circumstances (many of the drugs that save and alleviate the suffering of millions of lives every year were never tested in clinical trials). But in times of public health emergencies, such frailty in the understanding of how scientific breakthroughs often come about becomes especially harmful, as it discards valid alternative (scientific) approaches available to mitigate the effects of an unfolding crisis.

Randomized clinical trials (performed as early as six centuries ago [1]) are considered the gold standard to measure the efficacy of new drugs against specific disease endpoints. The wording 'specific' here is important. For example, vaccines must prime the immune system to recognize specific features of a given pathogen (e.g. the surface molecules of antigen-presenting cells) with a high degree of precision, inducing a very specific type of immune response needed for protection (otherwise they might be ineffective or induce non-antigen-specific responses that may lead to allergy and autoimmunity). Given this mode of action, clinical trials are, for now, the only way of testing vaccines. But many other therapies work in a very different way: they act in metabolic and microbiological webs that were recycled by evolutionary processes numerous times to fulfil different purposes. A chemical compound can affect several chemical pathways due to a common evolutionary origin, or simply because the same chemical is involved in different systems (e.g. [2]). This low specificity means that a large repertoire of existing therapies can be potentially recruited for other purposes. But how can these therapies be tested in a timely manner during an event such as a pandemic if evidence of benefit is constrained to expensive and time-consuming clinical trials? Fortunately, there is more to science. The possibility of recycling compounds for therapeutic uses other than those for which they were first discovered enables physicians and researchers around the globe to design potentially effective therapeutic solutions during a crisis. This mechanism makes use of a decentralized network that relies on multiple nodes of knowledge and clinical experience, which by relying on a large volume of small trials and rapid knowledge sharing increases the likelihood of insight and progressively favours the selection of solutions associated with positive individual results. This is far from being unscientific: knowledge is acquired through rigorous empirical observation, and independent replication, by the collective community. Confounding is possible with a small population, but becomes progressively less likely as results are replicated in multiple independent settings and demographic groups. There have been many voices in the scientific community complaining about the disorganization of this search for treatments during the Covid-19 pandemic [3]. Instead, the agility involved in this type of screening and selection of therapeutic solutions, as required during public health emergencies caused by novel pathogenic threats, should be encouraged.

\section{Antiviral use in the $2009 \mathrm{~A} / \mathrm{H} 1 \mathrm{~N} 1$ pandemic}

The A/H1N1 influenza strain originally detected in La Gloria, Mexico, in February 2009, was a novel mix of human, bird and swine genes. After causing an irregular number of deaths, the World Health Organization (WHO) 
declared the outbreak a pandemic in late April of that year (Rogers 2009). The US Centers for Disease Control and Prevention (CDC) estimates that from April 2009 to April 2010, this new influenza strain was responsible for approximately 61 million infections, 275,000 related hospitalizations and 12,500 deaths in the United States alone, with $87 \%$ of deaths occurring in individuals under the age of 65 [4].

Administrative guidelines around antiviral distribution were among the major controversies that arose during the crisis. Real-time studies varied in their recommendations, muddling discourse around antiviral usage. For instance, a review by Burch and collaborators [5] published in The Lancet Infectious Diseases warned against the use of antivirals in people presenting with symptoms, while another retrospective study using data from Argentina and Chile [6] suggested that increased antiviral use was, in fact, critical in decreasing transmission rates and in effectively reducing mortality rates in both ongoing and future influenza pandemics. Time proved that reluctance to antiviral use was unwise, as it resulted in the deaths of thousands of patients who could have been saved had prompt administration of what was a widely available pharmacological resource been utilized [7-10]. Retrospective analysis such as the systematic review by Pasquini-Descomps and collaborators [11] also highlighted the cost effectiveness of utilizing stockpiled antivirals.

At the time of the A/H1N1 outbreak, the CDC's official recommendation advised the use of antivirals only for "persons with suspected or confirmed influenza and who also met $>1$ of the following conditions: 1) illness that required hospitalization; 2) progressive, severe, or complicated illness, regardless of previous health status; and 3) risk for severe disease (e.g., patients with asthma, neurologic and neurodevelopmental conditions; chronic lung or heart disease; blood, endocrine, kidney, liver, and metabolic disorders; pregnancy; and those who were old or young)" [12]. The CDC maintained that their discretionary guidelines on the dissemination of antivirals would help ease the possibility of widespread antiviral resistance among the public [6]. Similarly, the WHO, which in the past had advocated for advance stockpiling of antivirals by government entities [13], also recommended treatment with oseltamivir only at advanced stages of the disease, namely "for patients who initially present with severe illness or whose condition begins to deteriorate [14]". However, it was already well known at the time that the treatment with antivirals was clinically meaningful at the onset of symptoms $[5,6,15,16]$. Since rapid pathogen clearance is critical in any infection where the risk of organ injury increases with time, an association between early antiviral treatment and superior clinical outcomes should not be surprising [10]. Furthermore, the argument that antiviral use could potentially lead to long-term antiviral resistance should have clearly been deprioritized, as in a pandemic situation any long-term implications would be comparatively small relative to the need to control a rapidly unfolding event.

Country authorities were faced with conflicting information about the antivirals: while they were told for years to stockpile antiviral resources to protect their population in the case of a pandemic, when that situation emerged, such a resource was regarded with official apprehension. It's no wonder that there was great variation in the way countries reacted to the $A /$ H1N1 pandemic. Those following the guidelines of the WHO were extremely conservative and almost reluctant in administering antivirals, advocating for administration only to those patients within the at-risk population. Other nations were more liberal in the administration of antivirals. Among the conservative responders were the United States, who in upholding CDC recommendations, used antivirals only among the clinically ill $[6,12]$ and Australia, who deployed limited use of their antiviral stockpiles [17]. Meanwhile, the United Kingdom pursued a more daring avenue, first using antiviral drugs for prophylaxis and then for treatment [18]. Likewise, in Japan, antiviral administration among pregnant women was stringently implemented, even before the WHO's 2009 inclusion of pregnant women within the risk group [19]. In comparisons across countries with similar socioeconomics, geographical location and demographics, those with the more liberal antiviral approach benefited from favorable health outcomes: Chile, which had an active antiviral usage policy during the entire course of the epidemic, saw a much lower mortality rate than Argentina, who reserved antiviral treatment for hospitalized patients [6]. In Mexico, higher survival rates were likewise associated with use of antiviral treatment [7].

\section{The case for patient choice in the SARS-CoV-2 pandemic}

Today's highly contested debates over the use protocols that include the repurposed use of drugs such as hydroxychloroquine (HCQ), chloroquine (CQ), corticosteroids, and other drugs in COVID-19-infected 
patients presents a similar dilemma to that experienced with antiviral usage in the 2009 A/H1N1 pandemic. The intent of this piece is not to delve into the details of potential COVID-19 treatments, their mechanisms of action or efficacy, but rather to highlight the lessons that should have been learnt from the previous pandemic crisis and the tradeoffs that come with a paralysing dependence on higher levels of scientific evidence during a rapidly evolving sanitary crisis.

Let us consider the case of $\mathrm{HCQ}$ and $\mathrm{CQ}$, two of the most prescribed drugs worldwide [20] given their effectiveness in the treatment and prevention of malaria [20], autoimmune diseases [21], as well as their activity against a range of bacterial, fungal and viral infections, including SARS-CoV-1 [22-25]. At the time of writing, published evidence towards the potential efficacy of HCQ as an early treatment for patients infected with SARSCoV-2 comes from in vitro studies showing its activity against this and other coronaviruses [26-28], from the study of its mode of action [23] and observational data on its effects during the early stage of COVID-19 disease when used in combination with other agents (e.g., zinc sulfate, azithromycin or doxycycline). A review from late May 2020, for instance, analyzed several studies, including two clinical trials, that have demonstrated significant outpatient treatment efficacy when HCQ was used in over 300,000 older adults with comorbidities [29]. Other studies have shown in-vivo evidence that zinc sulfate in combination with HCQ may play a role in therapeutic management for COVID-19 [30]. As with other zinc ionophores, HCQ may increase intracellular zinc levels to block viral replication. In India, sustained prophylaxis with HCQ by healthcare workers was associated with a reduction in the odds of getting infected [31]. A review of research papers published from January to April 2020, conducted by the Association of American Physicians and Surgeons, also concluded that the HCQ-AZ combination, when started immediately after diagnosis, appeared to be a safe and efficient treatment for COVID-19 [32]. As expected in an unfolding public health emergency, evidence has also come from reports of promising outcomes obtained by multiple treating physicians [3335]. As is inherent to observational research, it is certainly not possible to completely account for potential biases and confounding associated with the results of these studies. But although the efficacy of CQ and HCQ in COVID-19 treatment remains controversial, the possibility of benefit raised by these observations should tip risk-benefit assessments in favor of these approaches in the absence of other treatment options and of serious adverse effects when compared to those observed in palliative care alone.

Official guidelines are notoriously slow and conservative, hence hardly apt to adapt to a quickly developing crisis (in reaction to this situation, new initiatives that take advantage of the decentralized knowledge that emerges in clinical settings have been proposed [36]). In the case of HCQ, apprehension over its use has repeatedly emphasized the lack of confirmation of efficacy from randomized clinical trials. Despite the potential of repurposed drugs to curb disease evolution in the outpatient setting, guidelines for people with no evidence of oxygen requirement or pneumonia have frequently advised the use of supportive care alone [37]. Helping tip the balance towards the side of over-reluctance, protocols with HCQ have been widely misrepresented in the literature and media, with portrayals of lack of benefits based on administration alone or in hospital settings, to patients already in more advanced stages of the disease [35]. Concerns around potential side effects have also been widespread, despite the decades-long use of the drug and rare reports of toxicity [23]. Just recently, a study published by the Lancet resulted in the immediate halting of the administration of protocols including $\mathrm{HCQ}$ to millions of patients [38]. The study was soon later retracted.

Similar resistance was met over data from as early as March [39-41] suggesting the benefits of steroid drugs in combination with other agents to treat criticallyill COVID-19 patients - findings that were subsequently confirmed by physicians at the bedside $[40,42]$. As with the other drugs discussed, these are cheap, off-patent FDAapproved drugs that have been used for decades, with few side effects. Tragically, the WHO has recommended against the use of corticosteroids in COVID-19 [43]. The costs of this seemingly conservative approach by the WHO and other guideline issuing bodies in terms of lives lost are now becoming clearer: in late June, a large randomized controlled trial was published showing the benefits of a steroid drug (dexamethasone) in significantly reducing mortality in patients requiring oxygen or ventilator support [44]. Even now, some health officials are still reluctant about the use of the drug for Covid-19 patients due to its potential side effects [45].

History tends to highlight the damages of action, while downplaying the casualties of inaction. Side effects are easily attributed to pharmacological treatments in use, 
while deaths resulting from lack of treatment can always be attributed to the severity of the disease, complications or other deficiencies in health infra-estructures. This "paralysing perfectionism" is already patent in the development of antibiotics. Due to the strict regulatory environment, it costs between 800 million to 1 billion dollars to bring a new drug to market [46], no novel class of antibiotics has been developed in decades, with a substantial associated cost in terms of human lives lost. Efforts must be made to counter such information biases. One should not overlook the fact that the same antiviral that was withheld from the population during a pandemic of influenza, is now routinely used for seasonal influenza [47].

Though ideal, the world does not have the luxury of waiting for the results of clinical trials in the face of a highly transmissible new pathogen spreading swiftly across the globe. It is unforgivable that people were denied use of a widely available resource during the 2009 A/H1N1 influenza pandemic, leading to thousands of unnecessary deaths. The same reluctance towards the use of pharmacological protocols with early pre-clinical evidence of efficacy during both early and advanced disease stages of COVID-19 gives a sense of déjà vu to the current pandemic crisis. History is unfolding with striking resemblance. The main difference is that SARS-CoV-2 is both more deadly and infectious than the $\mathrm{A} / \mathrm{H} 1 \mathrm{~N} 1$ strain and the societal measures implemented to contain its spread have been more extreme, driving a surge of unemployment and poverty unprecedented in times of peace

When facing a novel and rapidly developing pathogenic threat, imposing tests of efficacy that require long periods of investigation automatically prevents the possibility of using virtually any tool. To make matters worse, even under a scenario where strong proof of efficacy is required before the use of interventions with potential side effects, such a strategy has been plagued with double standards. On the one hand, there has been extreme reluctance to use cheap and available off-the-shelf drugs with a long history of use in the population: before health professionals can recommend protocols including these same drugs to consenting patients infected with SARS-CoV-2, clinical trials are strongly suggested. On the other hand, non-pharmaceutical interventions such as social confinement are imposed on entire populations (consenting or not), although the efficacy of these measures on long-term health outcomes and public health in general has not been studied. We do this despite reasonable evidence to suggest that such measures can have harmful side effects, including a high morbidity and mortality burden, particularly among economically vulnerable populations. Such double standards might prove to be the greatest collective error of judgment in this generation.

As is the case with many societal conundrums, this is an instance wherein individual freedoms and responsibilities around the course of treatment should be respected. When conclusive answers are not available, but a major threat looms, patients should not have to wait until researchers come to a consensus. They must be informed about available options and their known risks, and be free to decide which course of treatment to pursue [48]. Evidence will come from the accumulated knowledge on the efficacy and side effects of existing drugs employed by physicians on the front line [35] and observational studies conducted while the crisis continues to unfold. We should learn from history, and avoid repeating the same mistakes.

Financial Suppor

This research received no specific grant from any funding agency, commercial or not-for-profit sectors.

Conflicts of Interest

None.

\section{REFERÊNCIAS}

1. Alonso WJ, Zhou SZ, Schuck-Paim C. The first clinical trial and controlled biological experiments. Hektoen International. 2016.

2. Fehér C, Soriano A, Mensa J. A Review of Experimental and Off-Label Therapies for Clostridium difficile Infection. Infectious diseases and therapy. 2017; 6: 1-35. http://dx.doi.org/10.1007/s40121-016-0140-z

3. Johnson CY. Chaotic search for coronavirus treatments undermines efforts, experts say. The Washington Post. 2020.

4. Shrestha SS, Swerdlow D, Borse RH, Prabhu VS, Finelli L. Estimating the burden of 2009 pandemic influenza $A(H 1 N 1)$ in the United States (April 2009-April 2010). Clin Infect Dis. 2011; 52 Suppl 1: S75-82. http://dx.doi.org/10.1093/cid/ciq012

5. Burch J, Corbett M, Stock C, Nicholson K, Elliot AJ, Duffy $S$. et al. Prescription of anti-influenza drugs for healthy adults: a systematic review and meta-analysis. The Lancet infectious diseases 2009; 9: 537-545. http://dx.doi.org/10.1016/S1473-3099(09)70199-9 
6. GoldsteinE, LipsitchM. Antiviral usageforH1N1 treatment: pros, cons and an argument for broader prescribing guidelines in the United States. PLoS currents. 2009; 1 : RRN1122. http://dx.doi.org/10.1371/currents.RRN1122

7. Chowell G. Impact of antiviral treatment and hospital admission delay on risk of death associated with 2009 A/H1N1 pandemic influenza in Mexico. BMC infectious diseases 2012; 12: 97.

8. Higuera IAL. Reducing occurrence and severity of pneumonia due to pandemic H1N1 2009 by early oseltamivir administration: a retrospective study in Mexico. PloS one. 2011; 6: e21838.

9. Hanshaoworakul W. Severe human influenza infections in Thailand: oseltamivir treatment and risk factors for fatal outcome. PloS one. 2009; 4: e6051.

10. Kumar A. Early versus late oseltamivir treatment in severely ill patients with 2009 pandemic influenza $A$ (H1N1): speed is life. J Antimicrob Chemother. 2011; 66: 959-963.

11. Pasquini-Descomps $H$, Brender $N$, Maradan D. Value for Money in H1N1 Influenza: A Systematic Review of the Cost-Effectiveness of Pandemic Interventions. Value in health: Value Health. 2017; 20: 819-827.

12. Atkins CY, Patel A, Taylor TH, Biggerstaff $M$, Mrlin $T L$, Dulin $S M$ et al. Estimating effect of antiviral drug use during pandemic (H1N1) 2009 outbreak, United States. Emerging infectious diseases 2011; 17: 15911598. http://dx.doi.org/10.3201 / eid1709.110295

13. Reddy D. Responding to pandemic (H1N1) 2009 influenza: the role of oseltamivir. J Antimicrob Chemother. 2010; 65 Suppl 2: ii35-ii40.

14. WHO. Global Alert and Response - Recommended use of antivirals. World Health Organization. World Health Organization, 2009(https://www. who.int/csr/disease/swineflu/notes/h1n1_use_ antivirals_20090820/en/). Accessed 23 June 2020.

15. Aoki FY, Macleod MD, Paggiaro O, Carewicz O, Sawy EL, Wat C. Early administration of oral oseltamivir increases the benefits of influenza treatment. The J Antimicrob. Chemother . 2003; 51: 123-129.
16. McClellan K, Perry CM. Oseltamivir: a review of its use in influenza. Drugs. 2001; 61: 263-283.

17. Commonwealth of Australia. ReviewofAustralia's health sectorresponseto pandemic(H1N1)2009: lessonsidentified. Department of Health and Ageing of Australia, 2011.

18. Monto AS, Preto S, Plotkin SA, Orentein WA. Response to the 2009 pandemic: effect on influenza control in wealthy and poor countries. Vaccine Elsevier, 2011; 29: http:// dx.doi.org/6427-6431. 10.1016/j.vaccine.2011.06.113

19. Nakai A, Saito S, Unno N, Kubo T, Minakami H. Review of the pandemic (H1N1) 2009 among pregnant Japanese women. J Obstet Gynaecol Re. 2012; 38: 757-762.

20. White NJ. The Treatment of Malaria. The New England journal of medicine Massachusetts Medical Society, 1996; 335: 800-806. http:// dx.doi.org/10.1111/j.1447-0756.2011.01812.x

21. Lee S-J, Silverman E, Bargman JM. The role of antimalarial agents in the treatment of SLE and lupus nephritis. Nature reviews. Nephrology nature. 2011; 7: 718-729.

22. Rolain J-M, Colson P, Raoult D. Recycling of chloroquine and its hydroxyl analogue to face bacterial, fungal and viral infections in the 21st century. Int J Antimicrob Agents. 2007;30: 297-308.

23. Devaux CA, Rolain JM, Colson, P, Raoult D. Newinsights on the antiviral effects of chloroquine against coronavirus: what to expect for COVID-19? Int J Antimicrob Agents 2020; 55: $\quad 105938 . \quad$ http://dx.doi. org/10.1016/j.ijantimicag. 2020.105938 24. Keyaerts $E$, et al. In vitro inhibition of severe acute respiratory syndrome coronavirus by chloroquine. Biochemical and biophysical research communications 2004; 323 (1): 264268. http://dx.doi.org/10.1016/j.bbrc.2004.08.085

25. Vincent MJ, Bergeron E, Benjannet S, Erickson BR, Rollin PE, Ksiazek TG. Chloroquine is a potent inhibitor of SARS coronavirus infection and spread. Virol J. 2005; 2: 69. http://dx.doi.org/10.1186/1743-422X-2-69

26. Liu J, Cao R, Xu M, Wang X, Zhang H, Hu H, 
et al. Hydroxychloroquine, a less toxic derivative of chloroquine, is effective in inhibiting SARSCoV-2 infection in vitro. Cell discovery. 2020; 6: 16. http://dx.doi.org/10.1038/s41421-020-0156-0

27. Hashem AM. Therapeutic use of chloroquine and hydroxychloroquine in COVID-19 and other viral infections: A narrative review. Travel medicine and infectious disease 2020; : 101735.

28. Yao X. In Vitro Antiviral Activity and Projection of Optimized Dosing Design of Hydroxychloroquine for the Treatment of Severe Acute Respiratory Syndrome Coronavirus 2 (SARS-CoV-2). Clinical infectious diseases: an official publication of the Infectious Diseases Society of America 2020; Published online: 9 March 2020. http://dx.doi.org/10.1093/cid/ciaa237.

29. Risch HA. Early Outpatient Treatment of Symptomatic, High-Risk Covid-19 Patients that Should be Ramped-Up Immediately as Key to the Pandemic Crisis. American journal of epidemiology 2020; Published online: 27 May 2020. http://dx.doi.org/10.1093/aje/kwaa093.

30. CarlucciP. Hydroxychloroquineandazithromycin pluszinc vs hydroxychloroquine and azithromycin alone: outcomes in hospitalized COVID-19 patients. medRxiv Cold Spring Harbor Laboratory Press. 2020; : 2020.05.02.20080036.

31. Chatterjee P. Healthcare workers \& SARS-CoV-2 infection in India: A case-control investigation in the time of COVID-19. Medknow Publications. 2020

32. AAPS. Sequential CQ / HCQ Research Papers and Reports January to April 20, 2020. Association of American Physicians and Surgeons, 2020 Apr.

33. Gautret P, Lagier JC, Parola P, Hoang VT, Meddeb L, Mailhe M. Hydroxychloroquine and azithromycin as a treatment of COVID-19: results of an open-label nonrandomized clinical trial. Internat J OfAntAg. 2020; 105949. https://dx.doi.org/10.1016/j.ijantimicag.2020.105949

34. Guérin V. Azithromycin and Hydroxychloroquine Accelerate Recovery of Outpatients with Mild/ Moderate COVID-19. Medic \& Phar. 2020.

35. Backman B. Doctor Zelenko Announces
CrowdProtocol. Youtube, 2020(https://youtu be/JfSRRhlto2g). Accessed 17 June 2020.

36. Adler N. Crowd Protocol seeks to end the pandemic. The Internet Protocol. 2020

37. Sanders JM. Pharmacologic Treatments for Coronavirus Disease 2019 (COVID-19): A Review. JAMA. 2020. http://dx.doi.org/10.1001/jama.2020.6019.

38. Mehra MR. RETRACTED: Hydroxychloroquine or chloroquine with or without a macrolide for treatment of COVID-19: a multinational registry analysis. The Lancet. 2020; http://dx.doi.org/10.1016/S0140-6736(20)31180-6.

39. Wu C. Risk Factors Associated With Acute Respiratory Distress Syndrome and Death in Patients With Coronavirus Disease 2019 Pneumonia in Wuhan, China. JAMA internal medicine. 2020. http:// dx.doi.org/10.1001/jamainternmed.2020.0994.

40. FLCCC Working Group. Treatment Protocol - Frontline COVID-19 Critical Care Working Group. FLCCC. (https://covid19criticalcare.com/ treatment-protocol/). Accessed 24 June 2020.

41. Wang Y. A retrospective cohort study of methylprednisolone therapy in severe patients with COVID-19 pneumonia. Signal trans and targ therapy. 2020.

42. Rolim C. Corticosteroidshavebeen used totreatCovid-19 in BrazilsinceMarch, saysimmunologist. Time24News.2020.

$\begin{array}{llr}\text { 43. WHO. Clinical management } & \text { of } \\ \text { Covid-19. World Health Organization, } 2020\end{array}$

44. Horby P. Effect of Dexamethasone in Hospitalized Patients with COVID-19: Preliminary Report. Medrxiv . 2020.

45. The Korea Herald. S. Korea cautious against dexamethasone's efficacy against new coronavirus. 2020.

46. Lobanovska M, Pilla G. Penicillin's Discovery and Antibiotic Resistance: Lessons for the Future? The Yale J of biology and M. 2017; 90: 135-145.

47. CDC. Influenza Antiviral Medications: Clinician Summary. Centers for Disease Control and Prevention. 2020. 
48. The Palmer Foundation. Another 10,000 doctors reinforce support for hydroxychloroquine against Covid-19, politics can not be above life. 2020. 